Ivan Franko State Pedagogical University of Drohobych (24, Ivan Franko Str., Drohobych 82100, Lviv region, Ukraine; e-mail: delenkonadia@mail.ru)

\title{
INFLUENCE OF ELECTRON-DEFORMATION EFFECTS ON THE ELECTRON STRUCTURE OF QUANTUM DOTS IN STRESSED NANOHETEROSYSTEMS
} PACS 73.21.La, 79.60.Jv

\begin{abstract}
In the framework of the self-consistent electron-deformation model, the theory describing the formation of the quantum potential band profile and the energy levels of an electron and a hole in a stressed nanoheterosystem with coherently-strained quantum dots has been developed, and their dependences on the degree of doping of the nanoheterosystem matrix and the quantum dot surface concentration have been analyzed. The character of the quantum potential in the nanoheterosystem is shown to be governed not only by the mechanical component of the electron-deformation potential, but also by the energy of electrostatic interaction between charges in a vicinity of the quantum dot-matrix interface, where the latter induces additional quasi-triangular potential barriers and wells.
\end{abstract}

Ke ywords: quantum dots, deformation, electron-deformation potential.

\section{Introduction}

Intensive researches of quasi-zero-dimensional semiconductor structures are stimulated by both the discovery of a number of essentially new fundamental phenomena and the wide opportunities of their application [1-3]. Quasi-zero-dimensional structures, quantum dots (QDs), with sizes $a \sim 1 \div 10^{2} \mathrm{~nm}$ are characterized by the confinement of charge carriers in all three space directions [4] and the emergence of the size quantization effect in their energy spectrum, at which the modification of the electronic properties of materials is the most pronounced. The discreteness in the energy spectrum of electrons and holes in QDs is used for the creation of optical nanolasers and other devices with a high thermal stability of the lasing frequency.

The optical and electronic properties of quasi-zerodimensional structures are governed, to a great extent, by the energy spectrum of spatially confined electrons and holes, which was calculated both making no allowance for the mechanical component of a

(c) R.M. PELESHCHAK, N.YA. KULYK, 2014

1098 deformation $[5,6]$ and making allowance for it in the framework of a rectangular quantizing potential [711]. The corresponding models considered the influence of a self-consistent deformation of the crystal matrix and QDs, the Laplace pressure at the QDmatrix interface, the finite size of the surrounding matrix, and the dependence of the parameter describing the mismatch between the contacting lattices at their heterointerface on the dimensions of a QD and the matrix. However, in those works, no allowance was made for the influence of the electrondeformation interaction. Hence, there emerges the necessity to develop a new theoretical model for a stressed nanoheterostructure with QDs and to consider a renormalization of the QD energy spectrum under the influence of the self-consistent electron-deformation interaction [12].

The difference between the lattice parameters of InAs and GaAs is considerable. In particular, the parameter mismatch amounts to $f=7 \%$ in the InAs/GaAs (001) nanoheterosystem with an array of QDs (InAs) and to $f=4 \%$ for $\mathrm{CdTe} / \mathrm{ZnTe}$. Therefore, the mechanical stresses that arise in a hetero-

ISSN 2071-0194. Ukr. J. Phys. 2014. Vol. 59, No. 11 
system with QDs substantially affect the structure of allowed bands and their discontinuity. In the case of coherently stressed QDs, provided that there are fields of elastic non-uniform stresses in their vicinity, and taking a self-consistent electron-deformation coupling into consideration, there emerges, besides the mechanical component of the deformation parameter, an electron-deformation one, which distinctly manifests itself in the formation of a quantizing potential at significant concentrations of conduction electrons $\left(n_{0} \leq 10^{18} \mathrm{~cm}^{-3}\right)$ in the nanoheterosystem matrix, which gives rise ultimately to variations in the positions of energy levels localized in a quantum dot. Therefore, in order to predictively control the spectral characteristics of nanooptoelectronic devices created on the basis of stressed nanoheterosystems with QDs (InAs/GaAs with InAs QDs and CdTe/ZnTe with CdTe QDs), it is necessary to know the regularities in modifications of the QD electron structure depending on the QD size, $R_{0}$, the average concentration of conduction electrons in the nanoheterosystem matrix, $n_{0}$, and the surface concentration of quantum dots, $N_{\mathrm{QD}}$. In this case, by changing the electron-deformation parameters - namely, the concentration of conduction electrons, $n_{0}$, the deformation potentials of the conduction and valence bands, and technological parameters (the surface concentration of quantum dots, $N_{\mathrm{QD}}$ ) - it is possible to retune the frequency of recombination radiation emitted at the electron transition from the localized electron level onto the hole one in a QD. In this connection, the problem concerning the influence of the self-consistent electron-deformation interaction on the electron structure of QDs in stressed nanoheterostructures becomes challenging.

This work aimed at calculating, in the framework of the model of self-consistent electron-deformation coupling, the spectrum of charge carriers in QDs renormalized by both the electron-deformation and electrostatic potentials at various concentrations of conduction electrons $n_{0}$ in the nanoheterosystem matrix and various surface concentrations of quantum dots $N_{\mathrm{QD}}$.

\section{Model of a Stressed Nanoheterosystem with Spherically Symmetric Quantum Dots}

In this work, we consider InAs/GaAs (CdTe/ZnTe) nanoheterosystems with ordered arrays of strained quantum dots without definite crystallographic orien-

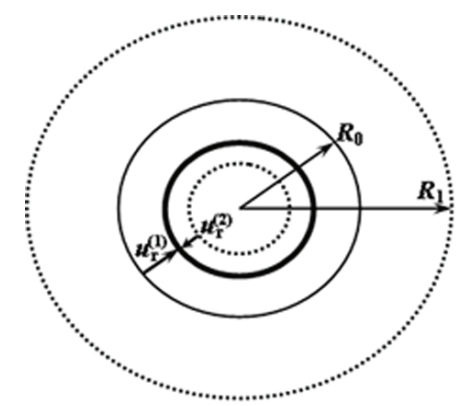

Fig. 1. Model of a spherically symmetric stressed quantum dot

tation, in particular, quantum dots with shapes that can be approximately regarded as spherically symmetric. For example, in the InAs/GaAs (001) heterosystem, such QDs are formed if the thickness of the grown InAs layer is about 2 monolayers $[13,14]$. Therefore, in what follows, the contribution made by island edges to the energy of elastic relaxation is neglected.

The ordered arrangement of stressed quantum dots on the crystalline matrix surface results from the elastic interaction between islands that arises owing to a mismatch between the InAs and GaAs lattice parameters. To reduce the problem with a considerable number of QDs to a problem with a single QD, the following approximation was made: the energy of pair elastic interaction between QDs is substituted by the energy of interaction between every QD and the averaged elastic deformation field created by all other QDs, $\sigma_{\text {ef }}(N-1)$.

The lattice constant in the grown InAs material $\left(a_{1}=6.08 \AA\right)$ is larger than that in the GaAs matrix $\left(a_{2}=5.65 \AA\right)$. Therefore, during the heteroepitaxial growth within the limits of the pseudomorphic growth of InAs on the GaAs layer, the InAs material undergoes a compressive deformation, and the GaAs matrix a stretching one. The formation of a stressed spherical nanoheterosystem takes place as follows: a spherical quantum dot of radius $R_{0}$ is regarded as an elastic spherical dilatational microinclusion (the solid thin curve in Fig. 1) located in a spherical cavity (the dotted curve in Fig. 1) in the GaAs matrix. The cavity volume is smaller than that of the microinclusion by $\Delta V$. Therefore, in order to insert the spherical microinclusion into the cavity, the former must be radially compressed by $u_{r}^{(1)}$, the radial component of the vector of mechanical displacement in the QD material, and the surrounding GaAs matrix 
must be radially stretched by $u_{r}^{(2)}$, the radial component of the vector of mechanical displacement in the matrix material. The result of the simultaneous action of deformations in the contacting nanomaterials is described by the volume variation $\Delta V=4 \pi f R_{0}{ }^{3}$ and the parameter of lattice mismatch between the contacting materials $f=\frac{a^{(1)}-a^{(2)}}{a^{(1)}} \approx 7 \%$.

Under the action of a non-uniform compressive deformation in the InAs (CdTe) QD material and a non-uniform stretching deformation in the GaAs (ZnTe) nanoheterosystem matrix, the band structure of the stressed nanoheterosystem with QDs locally changes. This modification, being a result of the selfconsistent electron-deformation coupling, gives rise to a redistribution of electrons in a vicinity of the quantum dot-matrix stressed interface. Accordingly, there emerges a non-uniform electrostatic potential both in the mechanically stressed QD and in the matrix, i.e. there appears an $n^{+}-n$ junction (the electrondeformation dipole $\mathbf{P}_{\text {el.-def. }}$ ) [15].

\section{Profile and Depth of Quantizing Potential for a Stressed Nanoheterosystem with Quantum Dots with regard for Self-Consistent Electron-Deformation Interaction}

The profile and the depth of a potential well for an electron in the stressed nanoheterosystem with QDs with regard for the contributions made by the electrostatic energy, $-e \phi^{(i)}(r)$, as well as the mechanical, $\varepsilon_{\text {mech. }}^{(i)}(r)$, and electron-deformation, $\varepsilon_{\text {el.-def. }}^{(i)}(r)$, components of the QD and matrix material deformations, are described by the following relation:

$$
\begin{aligned}
& \Delta V_{c}(r)=\Delta E_{c}(0)+a_{c}^{(2)}\left(\varepsilon_{\text {mech. }}^{(2)}(r)+\right. \\
& \left.+\varepsilon_{\text {el.-def. }}^{(2)}(r)\right)-a_{c}^{(1)}\left(\varepsilon_{\text {mech. }}^{(1)}(r)+\right. \\
& \left.+\varepsilon_{\text {el.-def. }}^{(1)}(r)\right)-e\left(\phi^{(2)}(r)-\phi^{(1)}(r)\right), \\
& i= \begin{cases}1 \equiv \text { InAs, } & 0 \leq r \leq R_{0}, \\
2 \equiv \text { GaAs, } & R_{0} \leq r \leq R_{1},\end{cases}
\end{aligned}
$$

where $R_{0}$ is the quantum dot radius, $R_{1}$ the matrix radius, $\Delta E_{c}(0)$ the potential well depth for the electron in the InAs quantum dot in the non-deformed InAs/GaAs heterostructure, and $a_{c}^{(1)}$ and $a_{c}^{(2)}$ are the constants of the hydrostatic deformation potential in the conduction and valence bands, respectively. The strain parameter for the $i$-th material in the nanoheterostructure,

$$
\varepsilon^{(i)}(r)=\operatorname{Sp} \varepsilon_{\text {mech. }}^{(i)}(r)+\operatorname{Sp} \varepsilon_{\text {el.-def. }}^{(i)}(r),
$$

where

$\operatorname{Sp} \varepsilon_{\text {mech. }}^{(i)}(r)=\varepsilon_{\text {rrmech. }}^{(i)}+\varepsilon_{\varphi \varphi \text { mech. }}^{(i)}+\varepsilon_{\theta \theta \text { mech. }}^{(i)}$,

is determined in terms of the atomic displacements $u_{r}^{(i)}$. They can be found from the equilibrium equation, which, in the case of spherical symmetry, looks like

$\frac{d^{2} u_{r}^{(i)}}{d r^{2}}+\frac{2}{r} \frac{d u_{r}^{(i)}}{d r}-\frac{2}{r^{2}} u_{r}^{(i)}=D^{(i)} e \frac{d \phi^{(i)}(r)}{d r}$

with the following boundary conditions $[11,16,17]$ :

$\left\{\begin{array}{l}4 \pi R_{0}^{2}\left(\left.u_{r}^{(2)}\right|_{r=R_{0}}-\left.u_{r}^{(1)}\right|_{r=R_{0}}\right)=\Delta V \\ \left.\sigma_{r r}^{(1)}\right|_{r=R_{0}}=\left.\sigma_{r r}^{(2)}\right|_{r=R_{0}}-P_{L}, \quad P_{L}=\frac{2 \alpha}{R_{0}} \\ \left.\sigma_{r r}^{(2)}\right|_{r=R_{1}}=-\sigma_{e f}(N-1)\end{array}\right.$

(the left-hand side of the first equation in system (3) is equal to the geometrical difference $\Delta V$ between the volumes of the microinclusion and the cavity in the GaAs matrix shown in Fig. 1),

$D^{(i)}=\frac{\left(1+\nu_{i}\right)\left(1-2 \nu_{i}\right)}{\left(a_{i}\right)^{3} E_{i}\left(1-\nu_{i}\right)}$,

$u_{r}^{(i)}$ is the radial component of an atomic displacement in the $i$-th semiconductor material, $a_{i}$ the lattice parameter of the $i$-th material in the nanoheterostructure, $\nu_{i}$ and $E_{i}$ are Poisson's ratios and the Young moduli of the QD and surrounding matrix materials, $e$ is the electron charge, $P_{L}$ the Laplace pressure, $\alpha$ the surface energy of a QD (InAs), $f$ the parameter of lattice mismatch between the contacting materials, and $\sigma_{r r}^{(i)}$ is the radial component of the mechanical stress tensor for the $i$-th material [18].

The general solution of the inhomogeneous equation (2) is sought as a sum of the mechanical and electron-deformation components of a displacement,

$u_{r}^{(i)}(r)=u_{r \text { mech. }}^{(i)}(r)+u_{\text {rel.-def. }}^{(i)}(r)$,

$$
u_{r \text { mech. }}^{(i)}(r)=C_{1}^{(i)} r+\frac{C_{2}^{(i)}}{r^{2}},
$$


$u_{\text {rel.-def. }}^{(i)}(r)=\frac{D^{(i)} e}{r^{2}} \int r^{\prime 2} \phi^{(i)}(r) d r^{\prime}$.

The field of mechanical displacements is determined by the following components of the tensor of mechanical strains in the QD and surrounding matrix materials:

$\varepsilon_{r r \text { mech. }}^{(1)}=\varepsilon_{\varphi \varphi \text { mech. }}^{(1)}=\varepsilon_{\theta \theta \text { mech. }}^{(1)}=C_{1}^{(1)}$

$\varepsilon_{\text {rrmech. }}^{(2)}=C_{1}^{(2)}-\frac{2 C_{2}^{(2)}}{r^{3}}$,

$\varepsilon_{\varphi \varphi \text { mech. }}^{(2)}=\varepsilon_{\theta \theta \text { mech. }}^{(2)}=C_{1}^{(2)}+\frac{C_{2}^{(2)}}{r^{3}}$.

The coefficients $C_{1}^{(1)}, C_{1}^{(2)}$, and $C_{2}^{(2)}$ are found from the solution of system (3) in view of expressions (4)(7) and (12), as well as expressions (20) and (21) for the electrostatic potential $\phi^{(i)}(r)$. The mechanical component of a uniform strain equals

$\varepsilon_{\text {mech. }}^{(i)}=\varepsilon_{r r \text { mech. }}^{(i)}+\varepsilon_{\varphi \varphi \text { mech. }}^{(i)}+\varepsilon_{\theta \theta \text { mech. }}^{(i)}=3 C_{1}^{(i)}$.

The field of electron-deformation displacements is described by the following components of the tensor of electron strains:

$\varepsilon_{r r \text { l. }- \text { def. }}^{(i)}=D^{(i)} e\left(\frac{2}{r^{3}} \int r^{\prime 2} \phi^{(i)}\left(r^{\prime}\right) d r^{\prime}-\phi^{(i)}\left(r^{\prime}\right)\right)$,

$\varepsilon_{\varphi \varphi \text { el.-def. }}^{(i)}=\varepsilon_{\theta \theta \text { el.-def. }}^{(i)}=$

$\left.=D^{(i)} e\left(\frac{1}{r^{3}} \int r^{\prime 2} \phi^{(i)}\left(r^{\prime}\right) d r^{\prime}\right)\right)$.

The electron-deformation component of the uniform strain equals

$\varepsilon_{\text {el.-def. }}^{(i)}=\varepsilon_{r r \text { el.-def. }}^{(i)}+\varepsilon_{\varphi \varphi \text { el.-def. }}^{(i)}+\varepsilon_{\theta \theta \text { el.-def. }}^{(i)}=$

$=D^{(i)} e \phi^{(i)}(r)$.

The mechanical stresses $\sigma_{r r}^{(1)}$ and $\sigma_{r r}^{(2)}$ in the quantum dot and matrix materials equal

$\sigma_{r r}^{(i)}=\frac{E_{i}}{\left(1+\nu_{i}\right)\left(1-2 \nu_{i}\right)} \times$

$\times\left[\left(1-\nu_{i}\right) \varepsilon_{r r}^{(i)}+\nu_{i}\left(\varepsilon_{\varphi \varphi}^{(i)}+\varepsilon_{\theta \theta}^{(i)}\right)\right]$.

The potential $\phi^{(i)}(r)$ is determined from the Poisson equation

$\Delta \phi^{(i)}(r)=\frac{e}{\varepsilon_{d}^{(i)} \varepsilon_{0}} \Delta n^{(i)}(r)$,

ISSN 2071-0194. Ukr. J. Phys. 2014. Vol. 59, No. 11 where $\varepsilon_{d}^{(i)}$ is the relative dielectric permittivity of the $i$-th material in the nanoheterosystem, $\Delta n^{(i)}(r)=$ $=n^{(i)}(r)-n_{0}$ is a variation of the electron concentration in a vicinity of the quantum dot-matrix heterointerface, which is determined in terms of the superposition of the products of wave functions,

$n^{(i)}(r)=\sum_{n} \frac{\psi_{n}^{*(i)}(r) \psi_{n}^{(i)}(r)}{\exp \left(\beta\left(\tilde{E}_{n}-\mu_{i}\right)\right)+1}$.

The wave functions are found from the Schrödinger equation

$\left[-\frac{\hbar^{2}}{2 m^{*(i)}} \Delta_{r}+\Delta V_{c}(r)\right] \psi_{n}^{(i)}(r)=E_{n} \psi_{n}^{(i)}(r)$

with the boundary conditions

$\left\{\begin{array}{l}\left.R_{n l}^{(1)}(r)\right|_{r=R_{0}}=\left.R_{n l}^{(2)}(r)\right|_{r=R_{0}}, \\ \left.\frac{1}{m^{*(1)}} \frac{d R_{n l}^{(1)}(r)}{d r}\right|_{r=R_{0}}=\left.\frac{1}{m^{*(2)}} \frac{d R_{n l}^{(2)}(r)}{d r}\right|_{r=R_{0}},\end{array}\right.$

where $m^{*(i)}$ is the effective electron mass in the $i$-th material, $E_{n}$ the energy of the $n$-th electron level in the quantum well, $\Delta V_{c}(r)$ the potential energy of electron (Eq. (1)), $n_{0}$ and $n^{(i)}(r)$ are the average concentration of conduction electrons and the concentration of charge carriers in the strained nanoheterostructure with the $\mathrm{QD}$, and $\mu_{i}$ is the chemical potential in the $i$-th material of nanoheterostructure, which is determined from the equation

$\frac{1}{\Omega_{0}} \int n(r) d r=n_{0}$,

where $\Omega_{0}$ is the elementary cell volume.

The solution of the Schrödinger equation (15) in the spherical coordinate system is sought in the form

$\psi_{n l m}^{(i)}(r, \theta, \phi)=R_{n l}^{(i)}(r) Y_{l m}^{(i)}(\theta, \phi)$,

where $Y_{l m}^{(i)}(\theta, \phi)$ are the spherical Legendre functions.

In the case of spherical symmetry, the Poisson equation (13) looks like

$\frac{d^{2} \phi^{(i)}}{d r^{2}}+\frac{2}{r} \frac{d \phi^{(i)}}{d r}=\frac{e}{\varepsilon_{d}^{(i)} \varepsilon_{0}}\left(n^{(i)}(r)-n_{0}\right)$.

The distributions of the charge carrier concentrations $n^{(i)}(r)$ in the quantum dot and in the matrix at

1101 
$T=0$ can be found by the formula

$$
\begin{aligned}
& n^{(i)}(r) \approx\left|\psi_{n l m}^{(i)}\right|^{2} \frac{N_{\mathrm{QD}}}{a_{i}} \times\left[\operatorname{erf}\left(\frac{\sqrt{2}}{\Delta E} E_{1}\right)+\right. \\
& +\operatorname{erf}\left(\frac{\sqrt{2}}{\Delta E}\left(\mu-E_{1}-\lambda_{0}^{(i)}-a_{c}^{(i)} \varepsilon_{r r}^{(i)}\right)\right)+ \\
& \left.+\sqrt{\frac{8}{\pi}} \frac{e}{\Delta E} \mathrm{e}^{\frac{-2\left(\mu-E_{1}-\lambda_{0}^{(i)}-a_{c}^{(i)} \varepsilon_{r r}^{(i)}\right)^{2}}{\Delta E^{2}}} e \phi^{(i)}(r)\right],
\end{aligned}
$$

where $N_{\mathrm{QD}}$ is the surface concentration of quantum dots, $\Delta E$ the Gaussian half-width, and $E_{1}$ the energy of the first localized electron level in the quantum well.

The solutions of the Poisson equation (18) in the quantum dot and in the matrix with regard for expression (19) for the electron concentration were determined, by using the averaged probability density $\left|\bar{\psi}^{(i)}\right|^{2}$. The following expressions were obtained:

$$
\begin{aligned}
& \phi^{(1)}(r)=A_{1} \frac{\sinh \left(\sqrt{\frac{1}{a_{1}}} r\right)}{r}-a_{1} b_{1}, \quad 0 \leq r \leq R_{0}, \\
& \phi^{(2)}(r)=B_{1} \frac{\exp \left(-\sqrt{\frac{1}{a_{2}}} r\right)}{r}+B_{2} \frac{\exp \left(\sqrt{\frac{1}{a_{2}}} r\right)}{r}- \\
& -a_{2} b_{2}-\frac{d_{2}}{2 r}\left[\exp \left(-\sqrt{\frac{1}{a_{2}}} r\right) E_{i}\left(\sqrt{\frac{1}{a_{2}}} r\right)+\right. \\
& \left.+\exp \left(\sqrt{\frac{1}{a_{2}}} r\right) E_{i}\left(-\sqrt{\frac{1}{a_{2}}} r\right)\right], \quad R_{0} \leq r \leq R_{1},
\end{aligned}
$$

where

$$
\begin{aligned}
& E_{i}(z)=-\int_{-z}^{\infty} t^{-1} \exp (-t) d t, \\
& \frac{1}{a_{1}}=\frac{e^{2}}{\varepsilon_{d}^{(1)} \varepsilon_{0} a_{1}}\left|\bar{\psi}^{(1)}\right|^{2} N_{\mathrm{QD}} \frac{\sqrt{\frac{8}{\pi}}}{\Delta E} \times \\
& \times \exp \left(\frac{-2\left(\mu-E_{1}-\lambda_{0}^{(1)}-a_{c}^{(1)} C_{1}^{(1)}\right)^{2}}{\Delta E^{2}}\right), \\
& \left.\frac{1}{a_{2}}=\frac{e^{2}}{\varepsilon_{d}^{(2)} \varepsilon_{0} a_{2}}\left|\bar{\psi}^{(2)}\right|^{2} N_{\mathrm{QD}} \frac{\sqrt{\frac{8}{\pi}}}{\Delta E} \times E^{(2)}-a_{c}^{(2)} C_{1}^{(2)}\right)^{2} \\
& \times \exp \left(\frac{-2\left(\mu-E_{1}-\lambda_{0}^{2}\right.}{\Delta E^{2}},\right.
\end{aligned}
$$

1102

$$
\begin{aligned}
& d_{2}=\frac{2 a_{c}^{(2)} C_{2}^{(2)} e}{\varepsilon_{d}^{(2)} \varepsilon_{0} a_{2}}\left|\bar{\psi}^{(2)}\right|^{2} N_{\mathrm{QD}} \frac{\sqrt{\frac{8}{\pi}}}{\Delta E} \times \\
& \times \exp \left(\frac{-2\left(\mu-E_{1}-\lambda_{0}^{(2)}-a_{c}^{(2)} C_{1}^{(2)}\right)^{2}}{\Delta E^{2}}\right), \\
& b_{1}=\frac{e}{\varepsilon_{d}^{(1)} \varepsilon_{0} a_{1}}\left|\bar{\psi}^{(1)}\right|^{2} N_{\mathrm{QD}}\left[\operatorname{erf}\left(\frac{\sqrt{2}}{\Delta E} E_{1}\right)+\right. \\
& \left.+\operatorname{erf}\left(\frac{\sqrt{2}}{\Delta E}\left(\mu-E_{1}-\lambda_{0}^{(1)}-a_{c}^{(1)} C_{1}^{(1)}\right)\right)-\frac{a_{1} n_{0}}{\left|\bar{\psi}^{(1)}\right|^{2} N_{\mathrm{QD}}}\right], \\
& b_{2}=\frac{e}{\varepsilon_{d}^{(2)} \varepsilon_{0} a_{2}}\left|\bar{\psi}^{(2)}\right|^{2} N_{\mathrm{QD}}\left[\operatorname{erf}\left(\frac{\sqrt{2}}{\Delta E} E_{1}\right)+\right. \\
& \left.+\operatorname{erf}\left(\frac{\sqrt{2}}{\Delta E}\left(\mu-E_{1}-\lambda_{0}^{(2)}-a_{c}^{(2)} C_{1}^{(2)}\right)\right)-\frac{a_{2} n_{0}}{\left|\bar{\psi}^{(2)}\right|^{2} N_{\mathrm{QD}}}\right] .
\end{aligned}
$$

The coefficients $A_{1}, B_{1}$, and $B_{2}$ in expressions (20) and (21) are determined from the matching conditions for the potentials $\phi^{(1)}(r)$ and $\phi^{(2)}(r)$ and for the normal components of the electric displacement vectors across the stressed heterointerface, as well as from the electroneutrality condition:

$$
\left\{\begin{array}{l}
\left.\phi^{(1)}(r)\right|_{r=R_{0}}=\left.\phi^{(2)}(r)\right|_{r=R_{0}}, \\
\left.\varepsilon^{(1)} \frac{d \phi^{(1)}(r)}{d r}\right|_{r=R_{0}}=\left.\varepsilon^{(2)} \frac{d \phi^{(2)}(r)}{d r}\right|_{r=R_{0}}, \\
\int_{0}^{R_{0}} r^{2} \Delta n^{(1)}(r) d r+\int_{R_{0}}^{R_{1}} r^{2} \Delta n^{(2)}(r) d r=0 .
\end{array}\right.
$$

Substituting the expressions obtained for the components of the mechanical and electron strain tensors (6)-(11) and expressions (20) and (21) for the electrostatic potential into formula (1), we obtain a formula for the potential energy of an electron in the stressed nanoheterosystem with quantum dots that involves the self-consistent electron-deformation interaction.

\section{Results of Calculations and Their Discussion}

The quantizing potential depth and the electron energy spectrum were calculated for the InAs/GaAs nanoheterosystem with the following parameters: $R_{0}=100 \AA, R_{1}=1000 \AA, a_{c}^{(1)}=-5.08 \mathrm{eV}$, $a_{c}^{(2)}=-7.17 \mathrm{eV}, a_{1}=6.08 \AA, a_{2}=5.65 \AA$, $m^{(1)}=0.057 m_{0}, m^{(2)}=0.065 m_{0}, \alpha=0.657 \mathrm{~N} / \mathrm{m}$,

ISSN 2071-0194. Ukr. J. Phys. 2014. Vol. 59, No. 11 
$n_{0}=10^{17} \div 10^{18} \mathrm{~cm}^{-3}, N_{\mathrm{QD}}=5.5 \times 10^{10} \mathrm{~cm}^{-2}$, and $\Delta E_{c}(0)=0.83 \mathrm{eV}$. The energy in the potential well and that of energy levels are reckoned from the top of the electron-deformation potential well bottom.

In Fig. 2, the results of numerical calculations for the dependence of the potential well depth $\Delta V_{c}(\mathbf{r})$ for an electron in the stressed QD on the quantum dot size are depicted. The contributions made by the electrostatic energy and the energy associated with a deformation of the QD and matrix materials are taken into account. One can see that, if the QD size $R_{0}$ grows, the depth of the electron quantizing potential increases monotonically, irrespective of whether the mechanical component of the electrondeformation potential is taken into account alone or together with the electrostatic potential. In particular, at the concentration of conduction electrons in the nanoheterosystem matrix $n_{0}=10^{17} \mathrm{~cm}^{-3}$ and the surface concentration of quantum dots $N_{\mathrm{QD}}=$ $=5.5 \times 10^{10} \mathrm{~cm}^{-2}$, the potential well depth amounts to 0.652 and $0.69 \mathrm{eV}$ for $R_{0}=30 \AA$ and $100 \AA$, respectively, if both the electron-deformation and electrostatic potentials are taken into account; in the other case, to $0.67 \mathrm{eV}$ and $0.692 \mathrm{eV}$, respectively. The reduction of the quantizing potential depth (Fig. 2, solid curve 2) with respect to the potential depth (Fig. 2, dotted curve 1) is associated with the contribution of the electrostatic energy and the action of an additional compression on the QD material that arises owing to the self-consistent electron-deformation component of the potential, $\varepsilon_{\text {el.-def. }}^{(i)}(r)$. It depends on the quantum dot size $R_{0}$, the degree of conduction band filling in the matrix, $n_{0}$, and the surface concentration of quantum dots, $N_{\mathrm{QD}}$.

In Figs. 3 and 4, the profiles and the depths of the quantizing potential for electrons in the InAs/GaAs nanoheterosystem with InAs quantum dots are shown for various concentrations of conduction electrons $\left(n_{0}=10^{17}\right.$ and $\left.10^{18} \mathrm{~cm}^{-3}\right)$ in the GaAs matrix with the surface QD concentration $N_{\mathrm{QD}}=5.5 \times 10^{10} \mathrm{~cm}^{-2}$. One can see that the electron-deformation component of the strain potential $\varepsilon_{\text {el.-def. }}^{(i)}(r)$ and the electrostatic energy $-e \phi^{(i)}(\mathbf{r})$ give rise to the energy shifts of the edges of allowed bands, $\Delta E_{c}^{(i)}=a_{c}^{(i)} \varepsilon^{(i)}(r)-e \phi^{(i)}(r)$. In particular, at $R_{0}=100 \AA, n_{0}=10^{17} \mathrm{~cm}^{-3}$, and $N_{\mathrm{QD}}=5.5 \times$ $\times 10^{10} \mathrm{~cm}^{-2}$, the quantum well bottom becomes nonuniformly shifted ("buckled") toward higher energies by about $45 \mathrm{meV}$ with respect to the deformed bot-

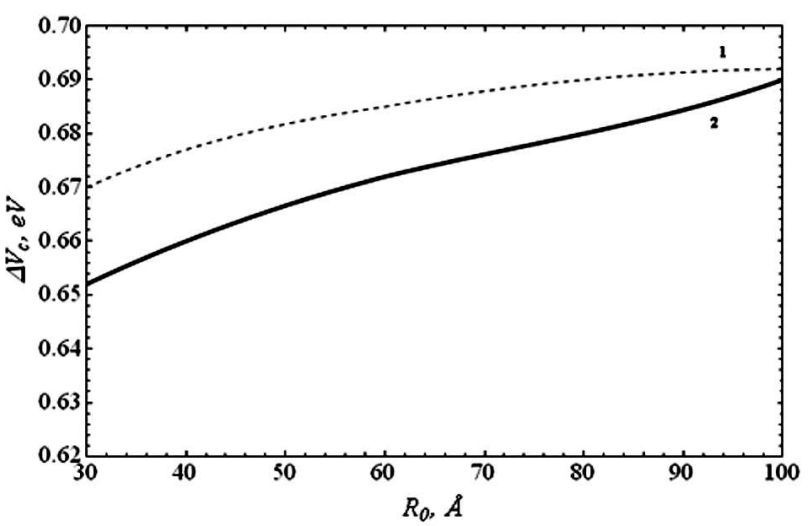

Fig. 2. Dependences of the potential well depth for electrons in a stressed spherical QDs on its radius $R_{0}$ at $N_{\mathrm{QD}}=$ $=5.5 \times 10^{10} \mathrm{~cm}^{-2}$ and $n_{0}=10^{17} \mathrm{~cm}^{-3}$ with regard for only the mechanical component of the electron-deformation potential (1, dotted curve) and both the electron-deformation and electrostatic potentials (2, solid curve)

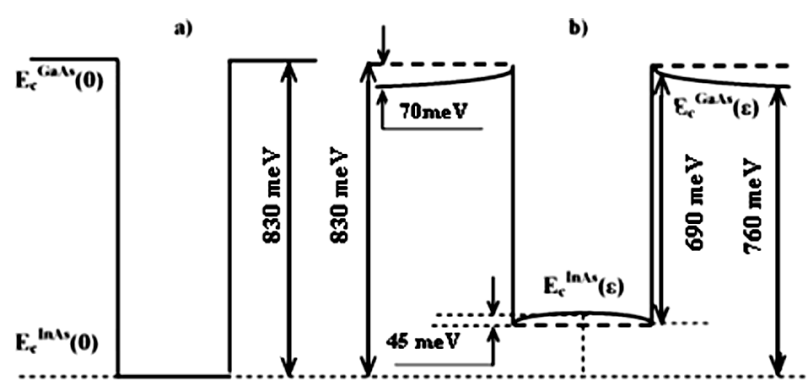

Fig. 3. Profiles of the quantizing potential in the InAs/GaAs nanoheterosystem with an InAs quantum dot of the radius $R_{0}=100 \AA$ and the electron concentration $n_{0}=10^{17} \mathrm{~cm}^{-3}$ that are formed $(a)$ owing to the break $\Delta E_{c}(0)$ of the conduction band only, ( $b$, dotted curve) owing to the break $\Delta E_{c}(0)$ of the conduction band and the mechanical component of the strain potential, and ( $b$, solid curve) owing to the break $\Delta E_{c}(0)$ of the conduction band, the mechanical and electrondeformation components of the strain potential, and the contribution of the electrostatic energy

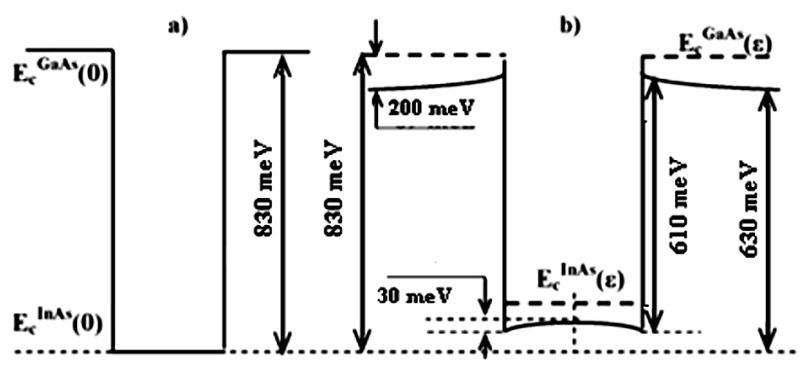

Fig. 4. The same as in Fig. 3, but for $n_{0}=10^{18} \mathrm{~cm}^{-3}$ 


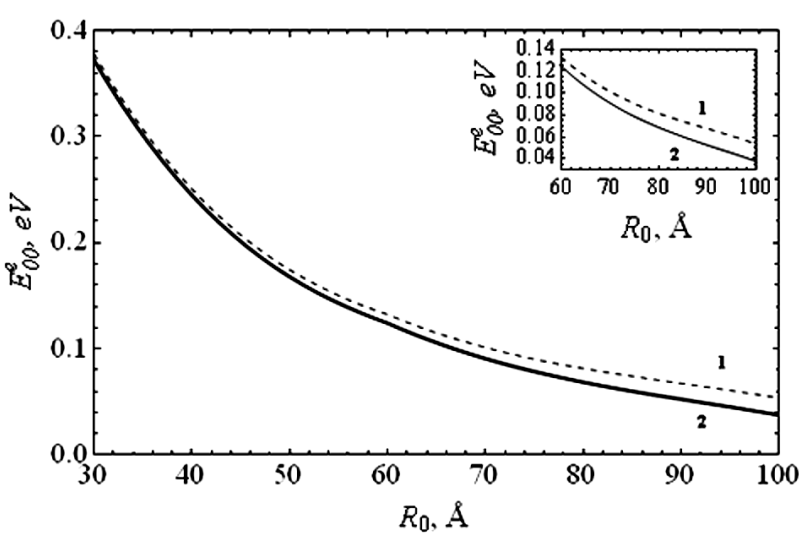

Fig. 5. Dependences of the electron ground state energy $E_{00}^{e}$ on the size of InAs quantum dots at $N_{\mathrm{QD}}=5.5 \times 10^{10} \mathrm{~cm}^{-2}$ and $n_{0}=10^{17} \mathrm{~cm}^{-3}$ with regard for only the mechanical component of the electron-deformation potential (1, dotted curve) and both the electron-deformation and electrostatic potentials (2, solid curve)

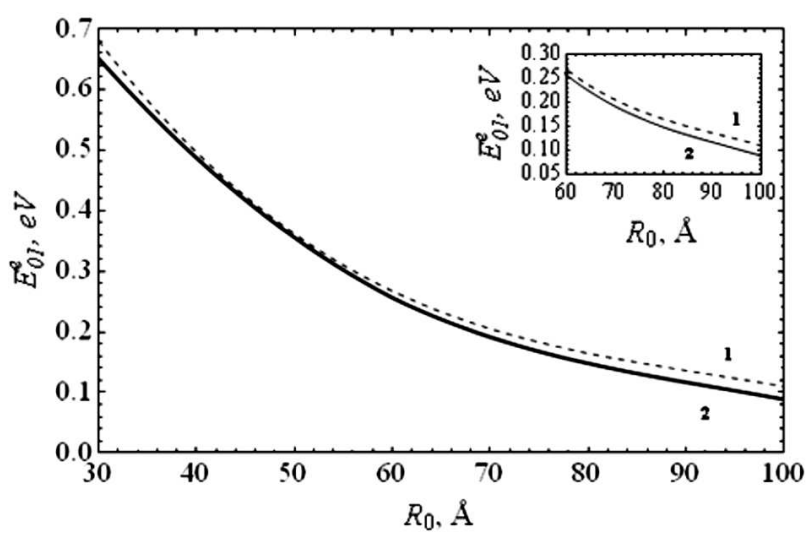

Fig. 6. The same as in Fig. 5, but for the electron excited state energy $E_{01}^{e}$

tom of the conduction band, which results from the mechanical deformation of the QD material, whereas the edges of allowed bands in the matrix become shifted toward lower energies by about $70 \mathrm{meV}$. If the average concentration of conduction electrons is an order of magnitude higher $\left(n_{0}=10^{18} \mathrm{~cm}^{-3}\right)$, the electron-deformation component of the strain potential gives rise to the decrease of allowed band edges in the matrix by $130 \mathrm{meV}$.

The electron energy spectra were calculated numerically, by using the shooting method [19] on the basis of the solution of the Schrödinger equation (15) with the quantizing potential (1) formed

\section{4}

by both the mechanical and electron components of the electron-deformation potential, $a_{c}^{(i)}\left(\varepsilon_{\text {mech. }}^{(i)}(r)+\right.$ $\left.+\varepsilon_{\text {el.-def. }}^{(i)}(r)\right)$, and the energy of electrostatic interaction between charges, $-e \phi^{(i)}(r)$, in a vicinity of the QD-matrix interface, which emerges owing to a redistribution of electrons induced by a non-uniform deformation of the QD and matrix materials.

In Figs. 5 and 6 , the results of numerical calculations for the dependence of the electron energy in the ground and excited states on the InAs QD size $R_{0}$ at $n_{0}=10^{17} \mathrm{~cm}^{-3}$ and $N_{\mathrm{QD}}=5.5 \times 10^{10} \mathrm{~cm}^{-2}$ are depicted. The comparison of curves 1 and 2 in Figs. 5 and 6 demonstrates that the electron-deformation component of the strain potential, $\varepsilon_{\text {el--def. }}^{(i)}(r)$, and the electrostatic energy $-e \phi^{(i)}(r)$ give rise to a nonmonotonic decrease of the electron energies in the ground and excited states with respect to the electron energy calculated taking only the mechanical compo-

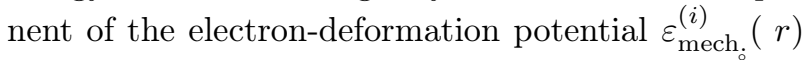
into account in the interval of $\mathrm{QD}$ sizes $30 \AA \leq$ $\leq R_{0} \leq 100 \AA$. In particular, the reduction of the energies of the ground and excited electron states is less pronounced (by $6 \mathrm{meV}$ ) in the QD size interval $30 \AA \leq R_{0} \leq 40 \AA$ than in the interval $60 \AA \leq R_{0} \leq 100 \AA$ (by $8-16 \mathrm{meV}$ ). Such a behavior of the electron energy levels in a QD depending on the QD size $R_{0}$ can be explained as follows. At larger $R_{0}$ (the QD becomes more massive), the influences of the surface (the Laplace pressure) and the uniform mechanical deformation of the crystal lattice in the QD material become weaker. As a result, the potential well depth increases. On the other hand, the electrostatic energy component of the quantizing potential stimulates the elevation of the conduction band bottom in the QD with respect to the mechanical component of the electron-deformation potential at $n_{0}=$ $=10^{17} \mathrm{~cm}^{-3}$ or its decrease at $n_{0}=10^{18} \mathrm{~cm}^{-3}$ (see Figs. 2 and 3 ).

As the average concentration of conduction electrons in the matrix material increases by an order of magnitude (to $n_{0}=10^{18} \mathrm{~cm}^{-3}$ ), the electron energy levels in the QD calculated with regard for the total electron-deformation and electrostatic potentials grow by $7 \mathrm{meV}$ at $R=100 \AA$, which is associated with an additional redistribution of electrons in the nanoheterosystem between the $\mathrm{QD}$ and the 
matrix. In addition, the increase of the surface QD concentration within the interval $3 \times 10^{10} \mathrm{~cm}^{-2} \leq$ $\leq N_{\mathrm{QD}} \leq 5.5 \times 10^{10} \mathrm{~cm}^{-2}$ provided the fixed average concentration of conduction electrons in the GaAs matrix, $n_{0}=10^{18} \mathrm{~cm}^{-3}$, results in a lowering of the electron energy levels in the QD, because the electron component of a strain increases with the average concentration of conduction electrons and additionally compresses the matrix and QD materials. As a result, the quantizing potential depth diminishes, and the electron energy levels become lower, respectively. In particular, at $R=$ $=100 \AA$, the energies of the ground and excited electron states in the QD amount to 44 and $98 \mathrm{meV}$, respectively, if $N_{\mathrm{QD}}=3 \times 10^{10} \mathrm{~cm}^{-2}$, and to 37 and $88 \mathrm{meV}$, respectively, if $N_{\mathrm{QD}}=5.5 \times$ $\times 10^{10} \mathrm{~cm}^{-2}$.

Hence, knowing the dependence of the energy level positions (renormalized by the electron-deformation and electrostatic potentials) in the QD on the quantum dot size $R_{0}$, the average concentration $n_{0}$ of conduction electrons in the nanoheterosystem matrix, and the surface concentration $N_{\mathrm{QD}}$ of quantum dots, it is possible to make a predictive control over the spectral characteristics of nanooptoelectronic devices (resonance tunnel diodes) created on the basis of stressed nanoheterosystems with QDs (InAs/GaAs with InAs QDs).

\section{Conclusions}

1. In the framework of the proposed model, the influence of the mechanical and electron-deformation components of the strain potential and the energy of electrostatic interaction of charges at the QD-matrix interface on the character of the quantizing potential in the stressed InAs/GaAs nanoheterosystem with InAs quantum dots is studied.

2. It is shown that the account for the electron component of the electron-deformation potential, $a_{c}^{(i)} \varepsilon_{\text {el.-def. }}^{(i)}(r)$, and the energy of electrostatic interaction between charges, $-e \phi^{(i)}(r)$, gives rise to the formation of additional quasitriangular barriers and quasitriangular potential wells in the quantizing potential, with their energy heights depending on both the concentration $n_{0}$ of conduction electrons in the matrix of the stressed nanoheterosystem and the surface concentration $N_{\mathrm{QD}}$ of QDs. Such stressed nanoheterosystems with QDs with the modified quan- tizing potential can be used as elements for the creation of resonance tunnel diodes, the transport properties of which can be controlled by varying the matrix doping level $n_{0}$ and the surface quantum dot concentration $N_{\text {QD }}$.

3. The electron component of electron-deformation potential and the component of electrostatic energy associated with the deformation in the QD and matrix materials are shown to lower the energies of the ground and excited electron states in QDs with the size $R=100 \AA$ by 16 and $21 \mathrm{meV}$, respectively.

1. N.N. Ledentsov, V.M. Ustinov, V.A. Shchukin et al., Fiz. Tekhn. Poluprovodn. 32, 385 (1998).

2. M. Tkach, V. Holovatsky, O. Voitsekhivska, M. Mykhalyova, and R. Fartushynsky, Phys. Status Solidi B 225, 331 (2001).

3. M. Korkusinski and P. Hawrylak, Phys. Rev. B. 63, 195311 (2001).

4. A.F. Tsatsulnikov and A.Yu. Egorov, Fiz. Tekh. Poluprovodn. 31, 851 (1997).

5. S.I. Pokutnyi, Ukr. J. Phys. Rev. 3, 46 (2006).

6. S.M. Reimann and M. Manninen, Rev. Mod. Phys. 74, 1283 (2002).

7. C. Pryor, J. Kim, L.W. Wang, A.J. Williamson, and A. Zunger, J. Appl. Phys. 83, 2548 (1998).

8. M. Grundmann, O. Stier, and D. Bimberg, Phys. Rev. B. 52, 11969 (1995).

9. A.V. Nenashev and A.V. Dvurechenskii, Zh. Eksp. Teor. Fiz. 118, 570 (2000).

10. V.V. Meshcheryakov, Fiz. Tverd. Tela 42, 1700 (2000).

11. B.V. Novikov, G.G. Zegrya, R.M. Peleshchak et al., Fiz. Tekhn. Poluprovodn. 36, 543 (2002).

12. I.V. Stasyuk and R.M. Peleshchak, Ukr. Fiz. Zh. 39, 856 (1994).

13. V.G. Talalaev, B.V. Novikov, S.Yu. Verbyn et al., Fiz. Tekh. Poluprovodn. 34, 467 (2000).

14. Z.M. Wang, K. Holmes, Yu.I. Mazur, and G.J. Salamo, Appl. Phys. Lett. 84, 1931 (2004).

15. R.M. Peleshchak and I.Ya. Bachynsky, Condens. Matter Phys. 12, 215 (2009).

16. V.P. Evtikhiev, O.V. Konstantinov, A.V. Matveentsev, and A.E. Romanov, Fiz. Tekh. Poluprovodn. 36, 79 (2002).

17. C. Teodosiu, Elastic Models of Crystal Defects (Springer, Berlin, 1982).

18. L.D. Landau and E.M. Lifshitz, Theory of Elasticity (Pergamon Press, New York, 1959).

19. M.V. Kutniv, Numerical Methods (Rastr-7, Lviv, 2010) (in Ukrainian).

Received 12.11.13

Translated from Ukrainian by O.I. Voitenko 
Р.М. Пелещак, Н.Я. Кулик

ВПЛИВ ЕЛЕКТРОН-ДЕФОРМАЦІЙНИХ ЕФЕКТІВ НА ЕЛЕКТРОННУ СТРУКТУРУ КВАНТОВИХ ТОЧОК У НАПРУЖЕНИХ НАНОГЕТЕРОСТРУКТУРАХ

$\mathrm{P}$ е $з$ ю м е

В межах самоузгодженої електрон-деформаційної моделі побудовано теорію формування зонного профілю квантуючого потенціалу та енергетичних рівнів електрона та дір- ки у напруженій наногетеросистемі з когерентно-напруженими квантовими точками залежно від ступеня легування $n$ матриці наногетеросистеми та їх поверхневої густини. Показано, що характер квантуючого потенціалу наногетеросистеми визначається не тільки механічною складовою електрон-деформаційного потенціалу, а також енергією електростатичної взаємодії зарядів в околі межі квантова точка - матриця, що приводять до утворення додаткових квазітрикутних бар'єрів та квазітрикутних потенціальних ям поблизу межі квантова точка - матриця. 